\title{
RECHERCHE ET NUMÉRATION DU COLI-BACILLE DANS LE LAIT \\ PAR LA MÉTHODE DU ROUGE NEUTRE
}

\author{
par THÉodor VASSILEFE \\ Docteur-Vétérìnaire.
}

(Travail du Lakoratoire d'Hygiène de la Faculté de Médecine et du Laboratoire des Industries animal ss de l'Ecole vétérinaire de Lyon.)

(Fin.)

LE BACTERIUM COLI DANS LE LAIT. .

\section{1. - SA Signification.}

1 Dans le lait cru. - On sait depuis longtemps que le lait de femme bien portante recueilli aseptiquement ne contient aucun microbe.

Du lait de vache recueilli, à l'exception des premiers jets, aussi aséptiquement que possible dans un récipient stérile, la traite étant faite sur des animaux bien pansés, avec des mains propres, après savonnage du pis et essuyage avec un linge propre, n'est pratiquement jamais, en masse, exempt de germes. Sa flore est peu variée. Elle est constituée de quelques microcoques, liquéfiants ou non, parfois de rares staphylocoques; le $B$. lactis acidis s'y rencontre aussi, mais pas toujours, et il est peu abondant.

Jamais on ne trouve de microorganismes du groupe Coli aerogenes. C'est postérieurement à la mulsion que ces derniers viennent ensemencer le lait quand les précautions les plus élémentaires ne sont pas prises ; ils sont apportés par les poussières du sol et des fourrages, par la malpropreté des récipients, celle de l'homme, celle del'animal, le mouillage avec des eaux contaminées.

Il est facile, cela étant dit, d'apprécier à sa juste valeur la signification du B. coli dans le lait cru.

Puisque le coli-bacille est un hôte constant du gros intestin de l'homme et des animaux domestiques, qu'il se trouve en abondance dans les exэréments de celui-là et de ceux-ci, sa présence dans le lait décèle à coup sûr la souillure de cet aliment par des matières excrémentitielles et, de plus, elle signifie la possibilité que le lait peut avoir véhiculé à un moment donné des germes pathogènes qui coexisteraient à côté du coli-bacille.

Quand l'analyse de l'eau permet d'y trouver le bacille typhique, le $B$. coli accompagne toujours ce dernier; mais l'inverse n'est pas fatalement vrai.

Une eau dans laquelle on décèle le $B$. coli est évidemment suspecte, mais elle ne contient pas obligatoirement le $B$. typhosus. $A$ fortiori, une eau sans $B$. coli est également sans $B$. typhique.

Ces considérations sont également d'application stricte au lait; 


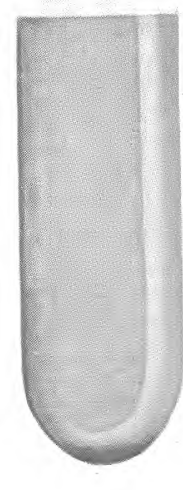

Réaclion posilive forte fluorescence
Réaclion posilive faible fluorescence

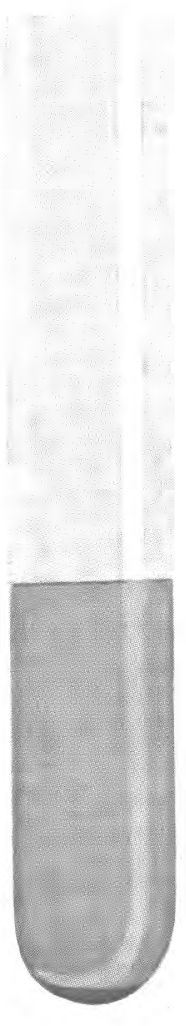

3

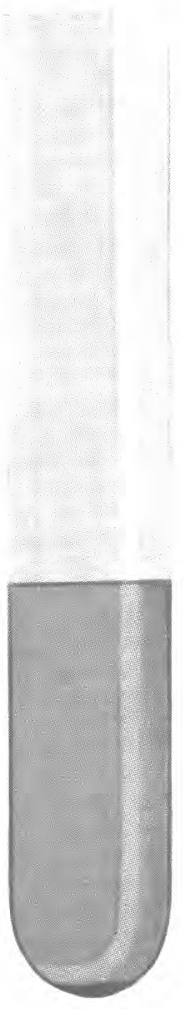

2

Réaction douleuse deuxième stade

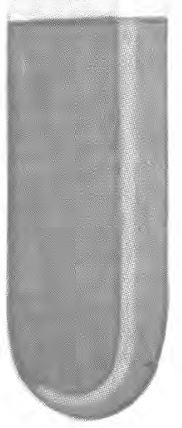

Bouillon non ensemencé
Réaction douleuse premier stade 
leur importance ne saurait échapper à l'hygiéniste soucieux de procurer du bon lait à l'enfance pour ses débuts.

Aussi la question de la contamination du lait par les matières fécales est-elle du plus haut intérêt, car si le lait est souillé, il est toujours suspect, en attendant qu'il puisse être dangereux.

A l'examen des échantillons de laits provenant des diverses régions qui approvisionnent Paris, BoRdas a trouvé chez certains d'entre eux un gramme de matières excrémentitielles parlitre et une richesse bactérienne de 20 millions de germes par centimètre cube.

On conçoit que de tels laits, si intensément souillés, d'une pareille richesse microbienne, véritables dilutions d'excréments, soient dangereux pour le fragile tractus gastro-intestinal des enfants et déclenchent brutalement chez ceux-ci des affections diarrhéiques à forme cholérique d'une extrême gravité. Et encore, nous ne parlons pas du risque, que nous serons tenté de dire presque fatal, que ces laits ont de contenir le bacille tuberculeux. Schroeder et Cotwon, dans des expériences célèbres, ont établi que la contamination du lait des bêtes réagissant à la tuberculine et qui ne présentent pas des lésions spécifiques mammaires pouvait souvent se faire indirectement par la chute dans le lait de matières excrémentitielles chargées de bacilles régurgités après des efforts de toux. Et quand on sait le danger de la tuberculisation de l'enfant par la voie digestive avec le bacille bovin, on peut à bon droit être effrayé des conséquenees tuberculigènes de la pollution du lait par les excréments.

Puisque, comme l'ont montré à KuFFerath les essais qu'il a faits, en Belgique, dans les vacheries du Comité National, à Nerom et à Boitsfort, on ne trouve jamais $d u$ B. coli dans les laits proprement traits, la recherche du coli-bacille s'impose dans l'examen des laits crus, afin d'en apprécier la valeur hygiénique.

20 Dans les laits pasteurisés. - La présence du coli-bacille dans le lait pasteurisé est généralement considérée comme une indication de chauffage insuffisant ou de réinfection après la pasteurisation.

Dans le but de vérifier les expériences de DE JoNG, qui avaient établi que le $B$. coli et le bacille tuberculeux sont plus résistants à la chaleur dans le lait que dans le bouillon et 1'eau, Axers et JoHnson ont chauffé des laits ensemencés de $B$. coli à $60^{\circ}$ pendant trente minutes : la moitié des souches résista, mais toutes furent tuées après chauffage à $65^{\circ} 6$ pendant trente minutes.

Toutefois, disent DE JoNG et DE GRAAFF, la certitude de tuer tous les coli-bacilles n'existe qu'avec une pasteurisation à 70-720 pendant trente minutes au moins.

Pour Whitraker HaLL, regrettant récemment la grande marge 
laissée aux Etats-Unis et au Canada dans l'interprétation légale du lait pasteurisé, la température de $62^{\circ}$ est pratiquement insuffisante.

Ainsi, le lait pasteurisé du commerce peut-il présenter des dangers analogues à ceux du lait cru, attendu que si, dans celui-ci les ferments lactiques inoffensifs empêchent les ferments nuisibles, dans le lait pasteurisé les ferments lactiques sont en partie tués en raison de la température à laquelle le lait est porté ; aussi, ce dernier n'est plus protégé contre la pullulation des bacilles à spores, dont certains (FLUGGE) sont capables de produire des toxines nuisibles.

$3^{\circ}$ Dans les laits stérilisés. - Naturellement, en raison des températures de la stérilisation, le B. coli n'y doit jamais subsister.

\section{2. - SA RECHERCHE.}

La recherche du B. coli dans le lait eomprend :

$1^{\circ}$ Des essais préliminaires ou essais de fermentation ;

$2^{\circ}$ Des recherches bactériologiques qualitatives et quantitatives.

10 Essais de fermentation. - Les ferments lactiques appartenant au groupe Coli provoquent presque toujours la formation de gaz ; ils agissent le plus souvent de façon très défavorable sur l'odeur et le goût du lait. On les décèle assez aisément, mais très grossièrement, par l'épreuve dite de fermentation (LöHNIS).

On verse dans de gros tubes à essais $50 \mathrm{~cm}^{3}$ de lait et on les porte au bainmarie à 40 degrés, On les examine entre douze à quarante-huit heures. Après douze heures, le la it recueilli proprement n'est pas modifié.

Le lait contenant du $B$. coli et certains autres germes d'origine exerémentitielle est coagulé. Le caillot, plus ou moins soulevé, est dilacéré, fragmenté par les gaz dégagés et collectés quelquefois sous forme de bulles assez grosses ; l'odeur n'est pas très agréable.

Au contraire, quand le lait coagule sous l'action des ferments lactiques normaux, son caillot est massif, non déchiqueté, homogène, ferme, gélatineux et l'odeur est agréable.

KUFFERATH admet que les laits contenant du $B$. coli et qui ne donnent pas de bulles de gaz à $37^{\circ}$ ne sont que faiblement contaminés.

$2^{\circ}$ Recherche bactériologique du coli-bacille dans le lait. Les procédés de recherche sont nombreux et généralement calqués sur ceux qui sont employés pour l'eau; cependant, ils ne conviennent qu'imparfaitement ; ils sont plus longs et moins exacts.

Les hygiénistes se préoccupent en ce moment d'établir la distinetion entre les coli-bacilles d'origine récente et ceux d'origine déjà aneienne que l'on peut rencontrer dans l'eau. En Amérique, les méthodes officielles comportent cette distinction.

En France, le Professeur Rochaix (1925), et son, élève Mile 
URTINetTe, en 1929, ont étudié la valeur des caractères proposés. Les coli-bacilles d’origine fécale récente présentent habituellement les trois caractères suivants :

Production abondante d'indol.

Réaction de Vos-Proskauer négative.

Réaction du rouge de méthyle positive.

Il ne semble pas que, pour le lait, la recherche de ces caractères ait un véritable intérêt. Cet aliment, très altérable, aliment exclusif de l'enfant et aliment principal du vieillard et de beaucoup de malades, doit être rigoureusement exempt de tout coli-bacille, quel qu'il soit, et ainsi, à l'abri de toute suspicion.

APPLICATION DE LA MÉTHODE DU ROUGE NEUTRE A LA RECHERCHE DU COLI-BACILLE DANS LE LAIT.

Les recherches de différents auteurs nous ont amenés à la conception d'une méthode de recherche rapide, au moyen du bouillon au rouge neutre, de la contamination du lait par les microbes provenant de matières d'origine intestinale, humaine ou animale.

Technique de la méthode. - Le bouillon au rouge neutre sera préparé de la façon suivante :

Dans un litre d'eau, on fait macérer 250 gr. de bouf (haché finement) pendant 24 heures; après, faire bouillir. Ecumer, et après refroidissement, on ajoute :

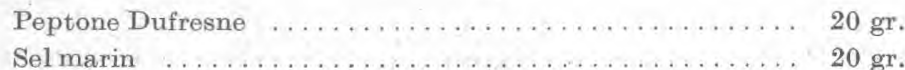

On ramène à $1.000 \mathrm{~cm}^{3}$ avee de l'eau, on fait bouillir de nouveau 5 minutes, puis, après refroidissement et repos, on filtre avec du papier de Chardin et on ajoute $10 \mathrm{em}^{3}$ de la solution suivante :

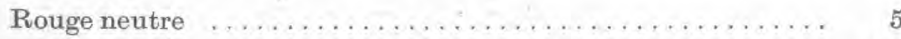

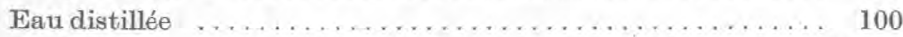

On porte à l'autoclave à $115^{\circ}$ pendant 30 minutes.

C'est en somme le bouillon de Savage, duquel on a supprimé la glucose.

Ce bouillon est réparti en une série de tubes de diverses grandeurs, pour faire l'échelle nécessaire à l'appréciation du degré de la contamination. Les quantités n'ont pas besoin d'être exactement mesurées.

L'échelle sera analogue à celle utilisée dans les méthodes ordinaires de dosage du coli-bacille.

La préparation du bouillon au rouge neutre a été exposée maintes fois, mais nous attirons l'attention sur les soins minutieux qui doivent être apportés dans sa préparation: Le rouge neutre doit être chimiquement pur (les rouges neutres de certaines firmes sont absolument inutilisables) et, dans la préparation du milieu, deux temps sont particulièrement délicats : la cuisson de la viande, qui doit être très 
peu poussée, et l'alcalinité, qui doit rester presque à la limite de la neutralité ou en être très voisine.

\section{RÉSULTATS OBTENUS.}

Nous avons examiné par la méthode du rouge neutre une trentaine de laits crus ou pasteurisés afin de pouvoir établir une technique permettant à l'avenir d'effectuer la numération, ou tout au moins de se rendre compte du degré de pollution du lait examiné.

Préalablement aux recherches bactériologiques nous nous sommes assuré des qualités des laits examinés pour savoir d'abord s'ils étaient crus ou pasteurisés, et aussi pour connaître leur degré de conservation.

La recherche de l'état de conservation du lait a été réalisée simplement par la prise de l'acidité avec le procédé Dornic (acidité D.).

Voici, résumée, la série de nos expériences :

Lats No 1. - Lait cru. Acidité : $19^{\circ} \mathrm{D}$.

Ce lait a été ensemencé dans le bouillon au rouge neutre préparé avec la solution à $5 \%$ de rouge neutre. Il n'a pas été dilué. Les quantités suivantes ont été utilisées :

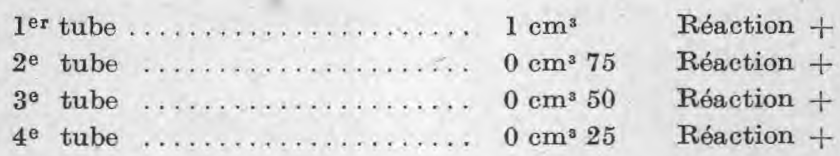

Manifestement, nos prises d'essai sont trop riches en microbes.

Pour la commodité du raisonnement, et bien que ce soit là une inexactitude, nous admettons que le virage est dû au coli-bacille seul. Nous admettons, d'autre part, qu'il suffit d'un seul individu bacillaire pour provoquer ledit virage, et nous pouvons alors affirmer que notre lait contient plus de :

$$
\frac{1.000}{0,25}=4.000 \text { coli-bacilles par litre. }
$$

Avec le même lait, nous avons ensemencé les mêmes quantités dans un bouillon préparé avec les mêmes doses d'une solution de rouge neutre à $10 \%$. Les résultats ont été les mêmes, c'est-à-dire :

$\begin{array}{ll}1 \mathrm{~cm}^{3} & \text { Réaction }+ \\ 0 \mathrm{~cm}^{3} 75 & \text { Réaction + } \\ 0 \mathrm{~cm}^{3} 50 & \text { Réaction + } \\ 0 \mathrm{~cm}^{3} 25 & \text { Réaction + }\end{array}$

La réaction ne semble donc pas être fonction très étroite de la concentration du rouge neutre dans le bouillon. C'est pourquoi nous adoptons, dans la suite; le bouillon classique avec la solution au rouge neutre à $5 \%$. 
LatT No 2. - Lait cru. Acidité : $25^{\circ} \mathrm{D}$. Ce lait a été dilué au 1/20, c'est-à-dire $1 \mathrm{~cm}^{3}$ de lait pour $19 \mathrm{~cm}^{3}$ d'eau stérilisée.

Les résultats de l'ensemencement ont été les suivants :

$$
\begin{aligned}
& 10 \mathrm{~cm}^{3} \quad \text { Virage et réaction + } \\
& 5 \mathrm{~cm}^{3} \quad \text { Virage et réaction + } \\
& 2 \mathrm{~cm}^{3} \frac{1}{2} \quad \text { Virage et réaction + } \\
& 1 \mathrm{~cm}^{3} \quad \text { Virage et réaction + }
\end{aligned}
$$

Notre dilution est done encore insuffisante, mais nous pouvons affirmer que le lait exảminé renferme plus de 20.000 coli-bacilles par litre, puisque $1 \mathrm{~cm}^{3}$ de dilution au 1/20 donne encore une réaction + .

Latr No 3. - Lait pasteurisé. Acidité : $17^{\circ} \mathrm{D}$. Ce lait a été dilué au 1/50, soit $1 \mathrm{~cm}^{3}$ de lait pour $49 \mathrm{~cm}^{3}$ d'eau stérilisée.

Deux séries d'essais ont été effectuées avec la dilution :

$$
\begin{aligned}
& 1^{\text {re }} \text { série : } 10 \mathrm{~cm}^{3} \quad \text { Virage }+ \\
& 5 \mathrm{~cm}^{3} \quad \text { Virage }+ \\
& 2 \mathrm{~cm}^{3} \quad \text { Virage }+ \\
& 1 \mathrm{~cm}^{3} \quad \text { Virage douteux } \\
& 2^{\mathrm{e}} \text { série : } 5 \mathrm{~cm}^{3} \quad \text { Virage }+ \\
& 2 \mathrm{~cm}^{3} 1 / 2 \text { Virage + } \\
& 1 \mathrm{~cm}^{3} \quad \text { Virage - } \\
& 1 / 2 \mathrm{~cm}^{3} \text { - Virage - }
\end{aligned}
$$

Ces deux séries donnent des résultats à peu près superposables et nous permettent de conclure que le lait renferme moins de 50.000 coli-bacilles par litre, mais plus de 25.000 .

LAIt No 4. - Lait cru. Acidité : 220. Dilué au 1/50.

Résultats de deux séries d'ensemencement :

$\begin{aligned} 1^{\text {re }} \text { série : } 5 \mathrm{~cm}^{3} & \text { Réaction + } \\ 2 \mathrm{~cm}^{3} 1 / 2 & \text { Réaction + } \\ 1 \mathrm{~cm}^{3} & \text { Réaction + } \\ 1 / 2 \mathrm{~cm}^{3} & \text { Réaction + } \\ 2^{\mathrm{e}} \text { série : } 2 \mathrm{~cm}^{3} 1 / 2 & \text { Réaction + } \\ 1 \mathrm{~cm}^{3} 1 / 4 & \text { Réaction + } \\ 1 / 2 \mathrm{~cm}^{3} & \text { Réaction + } \\ 1 / 4 \mathrm{~cm}^{3} & \text { Réaction + }\end{aligned}$

Ces résultats sont encore comparables et nous pouvons affirmer que le lait renferme au moins 200.000 coli-bacilles par litre.

LAIT No 5. - Lait pasteurisé. Acidité : $17^{\circ}$ D. Dilué au 1/50.

Résultats de l'ensemencement :

$\begin{aligned} 5 \mathrm{~cm}^{3} & \text { Réaction }+ \\ 2 \mathrm{~cm}^{3} 1 / 2 & \text { Réaction douteuse } \\ 1 \mathrm{~cm}^{3} & \text { Réaction - } \\ 1 / 2 \mathrm{~cm}^{3} & \text { Réaction - }\end{aligned}$

Le lait examiné renferme environ 20.000 coli-bacilles par litre. 
LaIT No 6, - Lait cru. Acidité : $18^{\circ} \mathrm{D}$.

On pratique un premier essai avec une dilution au 1/50:

$\begin{array}{cl}5 \mathrm{~cm}^{3} & \text { Réaction }+ \\ 2 \mathrm{~cm}^{3} 1 / 2 & \text { Réaction + } \\ 1 \mathrm{~cm}^{3} & \text { Réaction + } \\ 1 / 2 \mathrm{~cm}^{3} & \text { Réaction douteuse }\end{array}$

Le même lait est dilué au 1/100, et le nouvel essai donne :

$\begin{array}{ll}5 \mathrm{~cm}^{3} & \text { Réaction + } \\ 2 \mathrm{~cm}^{3} 1 / 2 & \text { Réaction + } \\ 1 \mathrm{~cm}^{3} & \text { Réaction douteuse } \\ 1 / 2 \mathrm{~cm}^{3} & \text { Réaction - }\end{array}$

Les deux essais sont encore comparables, ce qui justifie en quelque sorte la méthode, et nous pouvons affirmer que le lait contient environ 100.000 colibacilles au litre.

LAIT No 7. - Lait cru. Acidité : $21^{\circ} \mathrm{D}$.

- Première dilution à 1/200 donne les résultats suivarts :

$\begin{array}{cl}5 \mathrm{~cm}^{3} & \text { Réaction }+ \\ 2 \mathrm{~cm}^{3} 1 / 2 & \text { Réaction }+ \\ 1 \mathrm{~cm}^{3} & \text { Réaction }+ \\ 1 / 2 \mathrm{~cm}^{3} & \text { Réaction douteuse }\end{array}$

Deuxième dilution à $1 / 400$ donne les résultats suivarts :

$\begin{array}{cl}5 \mathrm{~cm}^{3} & \text { Réaction + } \\ 2 \mathrm{~cm}^{3} 1 / 2 & \text { Réaction + } \\ 1 \mathrm{~cm}^{3} & \text { Réaction douteuse } \\ 1 / 2 \mathrm{~cm}^{3} & \text { Réaction - }\end{array}$

Les résultats sont encore comparables et permettent d'attribuer une teneur en coli-bacilles approximative de 400.000 par litre de lait.

LATT No 8, - Lait pasteurisé. Acidité : $19^{\circ}$ D. Dilué au 1/50.

Résultats de l'ensemencement :

$\begin{array}{cl}5 \mathrm{~cm}^{3} & \text { Réaction }+ \\ 2 \mathrm{~cm}^{3} 1 / 2 & \text { Réaction + } \\ 1 \mathrm{~cm}^{3} & \text { Réaction + } \\ \frac{1}{1 / 2} \mathrm{~cm}^{3} & \text { Réaction douteuse } \\ 1 / 4 \mathrm{~cm}^{3} & \text { Réaction - }\end{array}$

Nous attribuons à ce lait une teneur approximative de 100.000 coli-bacilles par litre.

LaIt No 9. - Lait cru. Acidité : $20^{\circ}$ D. Dilué au 1/1.000.

Résultats de l'ensemencement :

$\begin{array}{cl}5 \mathrm{~cm}^{3} & \text { Réaction - } \\ 2 \mathrm{~cm}^{3} 1 / 2 & \text { Réaction - } \\ 1 \mathrm{~cm}^{3} & \text { Réaction - } \\ 1 / 2 \mathrm{~cm}^{3} & \text { Réaction - } \\ 1 / 4 \mathrm{~cm}^{3} & \text { Réaction - }\end{array}$

L'isolement ne nous a pas permis de mettre en évidence des bacilles. 
LATT No 10. - Lait pasteurisé. Acidité : $19^{\circ}$ D. Dilué au J/50.

Résultats de l'ensemencement :

$\begin{aligned} 5 \mathrm{~cm}^{3} & \text { Réaction + } \\ 2 \mathrm{~cm}^{3} 1 / 2 & \text { Réaction + } \\ 1 \mathrm{~cm}^{3} & \text { Réaction - } \\ 1 / 2 \mathrm{~cm}^{3} & \text { Réaction - } \\ 1 / 4 \mathrm{~cm}^{3} & \text { Réaction - }\end{aligned}$

Ce lait contient done une proportion de coli-bacilles comprise entre 20.000 et 50.000 par litre.

Latt No 11. - Lait cru. Ácidité : $19^{\circ} \mathrm{D}$.

Première dilution au 1/100:

$\begin{array}{ll}5 \mathrm{~cm}^{3} & \text { Réaction + } \\ 2 \mathrm{~cm}^{3} 1 / 2 & \text { Réaction + } \\ 1 \mathrm{~cm}^{3} & \text { Réaction + } \\ 1 / 2 \mathrm{~cm}^{3} & \text { Réaction + } \\ 1 / 4 \mathrm{~cm}^{3} & \text { Réaction + }\end{array}$

Deuxième dilution au 1/500:

$\begin{aligned} 5 \mathrm{~cm}^{3} & \text { Réaction }+ \\ 2 \mathrm{~cm}^{3} 1 / 2 & \text { Réaction }+ \\ 1 \mathrm{~cm}^{3} & \text { Réaction douteuse } \\ 1 / 2 \mathrm{~cm}^{3} & \text { Réaction - } \\ 1 / 4 \mathrm{~cm}^{3} & \text { Réaction - }\end{aligned}$

Les résultats fournis par la première série nous indiquent que le lait renferme plus de 400.000 coli-bacilles par litre. Ceux de la deuxième série nous montrent qu'en effet la teneur approximative est de 500.000 .

LAIT No 12. - Lait cru. Acidité : $18^{\circ} \mathrm{D}$.

Première dilution au 1/100:

$\begin{array}{cl}5 \mathrm{~cm}^{3} & \text { Réaction }+ \\ 2 \mathrm{~cm}^{3} 1 / 2 & \text { Réaction }+ \\ 1 \mathrm{~cm}^{3} & \text { Réaction }+ \\ 1 / 2 \mathrm{~cm}^{3} & \text { Réaction }+ \\ 1 / 4 \mathrm{~cm}^{3} & \text { Réaction douteuse }\end{array}$

Deuxième dilution au 1/500 :

$\begin{array}{cl}5 \mathrm{~cm}^{3} & \text { Réaction }+ \\ 2 \mathrm{~cm}^{3} 1 / 2 & \text { Réaction }+ \\ 1 \mathrm{~cm}^{3} & \text { Réaction douteuse } \\ 1 / 2 \mathrm{~cm}^{3} & \text { Réaction - } \\ 1 / 4 \mathrm{~cm}^{3} & \text { Réaction - }\end{array}$

Une petite différence existe dans les deux résultats : avec la première dilution, nous conclurions à 400.000 ; avec la deuxième dilution, à 500.000 coli-bacilles par litre. 
LaIt No 13. - Lait cru. Acidité : $20^{\circ}$ D. Dilué au 1/750.

$\begin{array}{cl}5 \mathrm{~cm}^{3} & \text { Réaction + } \\ 2 \mathrm{~cm}^{3} 1 / 2 & \text { Réaction + } \\ 1 \mathrm{~cm}^{3} & \text { Réaction + } \\ 1 / 2 \mathrm{~cm}^{3} & \text { Réaction - } \\ 1 / 4 \mathrm{~cm}^{3} & \text { Réaction - }\end{array}$

Le lait contient donc au moins 750.000 coli-bacilles par litre.

LaIt No 14. - Lait cru. Acidité : 29 D. Dilué au 1/1.000.

$\begin{array}{cl}5 \mathrm{~cm}^{3} & \text { Réaction + } \\ 2 \mathrm{~cm}^{3} 1 / 2 & \text { Réaction + } \\ 1 \mathrm{~cm}^{3} & \text { Réaction + } \\ 1 / 2 \mathrm{~cm}^{3} & \text { Réaction - } \\ 1 / 4 \mathrm{~cm}^{3} & \text { Réaction - }\end{array}$

Le lait contient done au moins 1 million de coli-bacilles par litre.

LatT No 15. - Lait cru. Acidité : 20 D. Dilué au 1/750.

$\begin{array}{cl}5 \mathrm{~cm}^{3} & \text { Réaction + } \\ 2 \mathrm{~cm}^{3} 1 / 2 & \text { Réaction + } \\ 1 \mathrm{~cm}^{3} & \text { Réaction + } \\ 1 / 2 \mathrm{~cm}^{3} & \text { Réaction - } \\ 1 / 4 \mathrm{~cm}^{3} & \text { Réaction - }\end{array}$

Le lait examiné renferme donc au moins 750.000 coli-bacilles par litre.

LaIt No 16. - Lait cru. Acidité : 18 D. Dilué au 1/750.

$\begin{array}{cl}5 \mathrm{~cm}^{3} & \text { Réaction + } \\ 2 \mathrm{~cm}^{3} 1 / 2 & \text { Réaction + } \\ 1 \mathrm{~cm}^{3} & \text { Réaction douteuse } \\ 1 / 2 \mathrm{~cm}^{3} & \text { Réaction - } \\ 1 / 4 \mathrm{~cm}^{3} & \text { Réaction - }\end{array}$

Le lait examiné renferme au plus 750.000 coli-bacilles par litre.

LATT No 17. - Lait pasteurisé. Acidité : 170 D. Dilué au 1/50.

$\begin{array}{cl}5 \mathrm{~cm}^{3} & \text { Réaction + } \\ 2 \mathrm{~cm}^{3} 1 / 2 & \text { Réaction douteuse } \\ 1 \mathrm{~cm}^{3} & \text { Réaction - } \\ 1 / 2 \mathrm{~cm}^{3} & \text { Réaction - } \\ 1 / 4 \mathrm{~cm}^{3} & \text { Réaction - }\end{array}$

Le lait examiné renferme au plus 20.000 coli-bacilles par litre.

LaIt No 18. - Lait cru. Acidité : 20 D. Dilué au 1/750.

$\begin{array}{cl}5 \mathrm{~cm}^{3} & \text { Réaction + } \\ 2 \mathrm{~cm}^{3} 1 / 2 & \text { Réaction }+ \\ 1 \mathrm{~cm}^{3} & \text { Réaction + } \\ 1 / 2 \mathrm{~cm}^{3} & \text { Réaction }+ \\ 1 / 4 \mathrm{~cm}^{3} & \text { Réaction douteuse }\end{array}$

Le lait examiné renferme au plus 3 millions de coli-bacilles par litre. 
LATT No 19. - Lait cru. Acidité : $19^{\circ} 5$ D. Dilué au 1/750.

$\begin{array}{cl}5 \mathrm{~cm}^{3} & \text { Réaction + } \\ 2 \mathrm{~cm}^{3} 1 / 2 & \text { Réaction + } \\ 1 \mathrm{~cm}^{3} & \text { Réaction + } \\ 1 / 2 \mathrm{~cm}^{3} & \text { Réaction douteuse } \\ 1 / 4 \mathrm{~cm}^{3} & \text { Réaction - }\end{array}$

- Le lait examiné renferme au plus 1.500.000 coli-bacilles par litre.

LAIT No 20. - Lait cru. Acidité : 41ํD. Dilué au 1/2.000.

$\begin{array}{cl}10 \mathrm{~cm}^{3} & \text { Réaction + } \\ 5 \mathrm{~cm}^{3} & \text { Réaction + } \\ 2 \mathrm{~cm}^{3} 1 / 2 & \text { Réaction + } \\ 1 \mathrm{~cm}^{3} & \text { Réaction + } \\ 1 / 2 \mathrm{~cm}^{3} & \text { Réaction + } \\ 1 / 4 \mathrm{~cm}^{3} & \text { Réaction - }\end{array}$

Le lait examiné, qui est en état d'altération avancée, renferme au moins 4 millions de coli-bacilles par litre.

Latt No 21. - Lait cru. Acidité : 20 D. Dilué au 1/1.000.

$\begin{array}{cl}5 \mathrm{~cm}^{3} & \text { Réaction }+ \\ -2 \mathrm{~cm}^{3} 1 / 2 & \text { Réaction }+ \\ 1 \mathrm{~cm}^{3} & \text { Réaction }+ \\ 1 / 2 \mathrm{~cm}^{3} & \text { Réaction }+ \\ 1 / 4 \mathrm{~cm}^{3} & \text { Réaction douteuse }\end{array}$

Le lait examiné renferme au plus 4 millions de coli-bacilles par litre.

LATt No 22. - Lait cru. Acidité : 19 D. Dilué au 1/1.000.

$\begin{array}{cl}5 \mathrm{~cm}^{3} & \text { Réaction + } \\ 2 \mathrm{~cm}^{3} 1 / 2 & \text { Réaction + } \\ 1 \mathrm{~cm}^{3} & \text { Réaction + } \\ 1 / 2 \mathrm{~cm}^{3} & \text { Réaction + } \\ 1 / 4 \mathrm{~cm}^{3} & \text { Réaction - }\end{array}$

Le lait examiné renferme au moins 2 millions de coli-bacilles par litre.

LAIT No 23. - Lait cru. Acidité : 19 D. Dilué au 1/1.000.

$\begin{array}{cl}5 \mathrm{~cm}^{3} & \text { Réaction + } \\ 2 \mathrm{~cm}^{3} 1 / 2 & \text { Réaction + } \\ 1 \mathrm{~cm}^{3} & \text { Réaction + } \\ 1 / 2 \mathrm{~cm}^{3} & \text { Réaction + } \\ 1 / 4 \mathrm{~cm}^{3} & \text { Réaction + }\end{array}$

Le lait examiné renferme done plus de 4 millions de coli-bacilles par litre.

LAIT No 24. - Lait cru. Acidité : $30^{\circ}$ D. Dilué au 1/1.500.

$\begin{aligned} 5 \mathrm{~cm}^{3} & \text { Réaction + } \\ 2 \mathrm{~cm}^{3} 1 / 2 & \text { Réaction + } \\ 1 \mathrm{~cm}^{3} & \text { Réaction douteuse } \\ 1 / 2 \mathrm{~cm}^{3} & \text { Réaction - } \\ 1 / 4 \mathrm{~cm}^{3} & \text { Réaction - } \\ 1 / 5 \mathrm{~cm}^{3} & \text { Réaction - }\end{aligned}$

Le lait examiné renferme done au plus 1.500.000 mille coli-bacilles par litre. 
LaIt No 25, - Lait cru. Acidité : 27으. Dilué au 1/1.500.

$\begin{array}{cl}5 \mathrm{~cm}^{3} & \text { Réaction + } \\ 2 \mathrm{~cm}^{3} 1 / 2 & \text { Réaction + } \\ 1 \mathrm{~cm}^{3} & \text { Réaction + } \\ 1 / 2 \mathrm{~cm}^{3} & \text { Réaction douteuse } \\ 1 / 4 \mathrm{~cm}^{3} & \text { Réaction - } \\ 1 / 5 \mathrm{~cm}^{3} & \text { Réaction - }\end{array}$

Le lait examiné renferme done au plus 3 millions de coli-bacilles par litre.

\section{INTERPRÉTATION DES RÉSULTATS.}

Nous arrêtons là la liste des laits qui ont été examinés par la méthode au rouge neutre, bien qu'une quinzaine d'autres échantillons aient été examinés. Mais comme, d'une part, ils n'apportaient rien de plus à l'appui de notre thèse, et que, d'autre part, nous n'avons effectué d'isolement ultérieurs que sur les 25 échantillons ci-dessus, il nous a paru plus sûr de ne tenir compte que d'eux dans ce travail.

A l'examen des réactions obtenues, quelques réflexions s'imposent tout d'abord: la quantité de microbes attaquant le rouge neutre est extrêmement variable suivant les laits examinés et, d'une façon générale, elle est beaucoup plus grande dans les laits crus que dans les laits pasteurisés, ce qui n'a évidemment rien qui puisse nous surprendre.

De plus, on ne peut établir de relations, particulièrement étroites, entre l'acidité d'un lait et sa teneur en microbes actifs sur le rouge neutre.

Enfin, dans la traduction de nos résultats expérimentaux, nous avons évidemment donné des chiffres, mais, il faut bien l'avouer, ils ne présentent pas une précision mathématique; du moins, ils permettent une comparaison plus facile.

Il doit également être entendu que si pour la commodité de l'expression des résultats, nous n'avons parlé que de coli-bacilles, d'autres microbes - également actifs sur le rouge neutre et dont la signification dans les eaux comme dans le lait est la même que celle du coli-bacille - ont pu également intervenir dans nos recherches. Qu'avons-nous, en effet, recherché dans nos expériences ? L'établissement d'une méthode de contrôle suffisamment précise et rapide qui nous permette d'évaluer le degré de pollution d'un lait de marché, surtout d'une pollution par les matières excrémentitielles. Il nous semble que, comme pour les eaux, la méthode au rouge neutre est susceptible de rendre des services et que nous sommes arrivé au résultat cherché, à condition de préconiser dès maintenant et à la lumière de nos expériences, une technique d'application.

Technique proposée. - Nous ne reviendrons pas sur la prépara- 
tion du milieu de culture. La question a déjà été traitée et nous nous bornerons à indiquer les précautions à prendre pourl'ensemencement.

Il est indiqué de rechercher, d'abord, comme nous l'avons fait, si le lait à examiner est cru ou pasteurisé ; puis, on détermine l'acidité du lait.

Enfin, le lait doit être dilué assez fortement, car, contrairement à l'eau, il est en général - et nos expériences le montrent - extrêmement riche en microbes. De plus, si on ensemençait dans le bouillon du lait en nature, on introduirait d'assez grosses quantités de substances nouvelles - et notamment du lactose et dela caséine, qui pourraient tout au moins modifier dans une certaine mesure les résultats.

Les règles générales de dilution qui résultent de nos expériences sont les suivantes:

\section{Pour le lait pasteurisé :}

a) Si l'acidité est comprise entre $17^{\circ}$ et $19^{\circ}$, la dilution au 1/50 nous paraît suffisante.

b) Si l'acidité est comprise entre $19^{\circ}$ et $25^{\circ}$, diluer à $1 / 100$.

2 o Pour le lait cru :

a) Si l'acidité est comprise entre $17^{\circ}$ et $19^{\circ}$, une dilution à $1 / 500$ sera suffisante.

b) Entre $19^{\circ}$ et $25^{\circ}$, diluer à $1 / 1.000$.

c) Entre $25^{\circ}$ et $30^{\circ}$, diluer à $1 / 1.500$.

d) Entre $30^{\circ}$ et $40^{\circ}$, diluer à $1 / 2.000$.

L'ensemencement aura lieu avec les quantités de dilution que nous avons utilisées, soit respectivement $5 \mathrm{~cm}^{3}, 2 \mathrm{~cm}^{3} 1 / 2,1 \mathrm{~cm}^{3}$, $1 / 2 \mathrm{~cm}^{3}$ et $1 / 4 \mathrm{~cm}^{3}$. On pourra même resserrer les intervalles ou préparer plusieurs séries, ce qui a l'avantage de permettre un contrôle.

L'examen des résultats de la réaction pourra avoir lieu au bout de 24 heures d'étuve à $41^{\circ} 5$, ce qui suffit bien dans tous les cas. Sur la planche colorée jointe, on peut se rendre compte des différences très nettes que présentent le milieu de culture non ensemencé (tube no 1 ), les réactions plus ou moins douteuses (tubes $n^{\circ} 2$ et $n^{\circ} 3$ ) et enfin la réaction positive (tube $n^{0} 4$ ) et fortement positive (tube $n^{0} 5$ ).

Nous insistons encore sur le fait que doivent seules être comptées comme réactions positives celles qui s'accompagnent à la fois du virage au jaune canari et de la fluorescence.

En résumé, si l'on tient compte des précautions que nous venons de signaler et si on applique scrupuleusement la technique indiquée on doit obtenir des résultats qui concordent avec ceux que nous avons nous-même signalés. 


\section{CONCLUSIONS.}

I. - La méthode au rouge neutre a pris rang, depuis une vingtaine d'années, parmi les moyens de recherches rapides du coli-bacille dans l'eau de boisson.

II. - La méthode n'a jamais été tentée aveo succès dans les recherches du coli-bacille dans le lait.

III. - D'après les expériences qui précèdent, nous croyons être en droit d'afffirmer que :

$1^{\circ} \mathrm{Du}$ lait contenant des coli-bacilles ensemencé en bouillon au rouge neutre, mis à l'étuve à $41^{\circ} 5$, donne, dans les 24 heures, un virage au jaune canari avec fluoreseence verte.

$2^{\circ}$ La réaction au jaune canari avec fluorescence verte est constante, rapide et présente réellement peu de eauses d'erreur.

$3^{\circ}$ Cette réaction est très sensible.

IV. - La réaction est due à la réduction du rouge neutre en milieu alcalin (ammoniaque).

V. - Les sucres gênent la réaction. Il est indispensable de les supprimer dans la composition du milieu (Rocharx).

VI. - Il en est de même de la présence del'oxygène de l'air. Aussi, est-il préférable de faire les cultures en l'absence de l'air, commel'ont proposé FABRE-DOMERGUE et LEGENDRE.

VII. - En se plaçant dans toutes les conditions énumérées, on réduira au minimum le nombre des erreurs. Malgré tout, on ne devra jamais affirmer la présence du coli-bacille dans le lait sans l'avoir isolé et en avoir établi les caractères culturaux, biochimiques et les caractères de coloration.

VIII. - La teneur du lait pasteurisé en coli-bacilles est considérablement moindre que celle du lait cru.

IX. - Pour les raisons que nous avons indiquées, la recherche des caractères différenciant les coli-bacilles d'origine fécale récente des autres, ne présente pas de véritable intérêt pour le lait.

\section{BIBLIOGRAPHIE.}

S. Henry A yers et Ir. W. T. Johnson. La résistance du coli-bacille à la pasteurisation. Journal of Agricultural Research, Department of Agriculture, vol.III, no 5, 15 février 1915, pp. 401-410. Le Lait, 1926, p. 367.

BrAUN. Le rouge neutre et le diagnostic de la souillure des eaux de boisson par le coli-bacille. Bulletin de l'Institut Pasteur, t. IV, n 13, 15 juillet 1906.

Chantemesse et Widar. Différenciation du B. typhique et du Coli communis. C. R. de l'Acad. des Sciences, 9 novembre 1889.

L. Camus. Des "tests " microbiologiques de contamination dans le contrôle du lait. Thèse de Lyon, 1921. 
Codrmont. Précis de Bactériologie, $5^{\ominus}$ édition, p. 434.

Courmont et Rochatx. Précis d'Hygiène, Masson, 1921.

Den Dooren de Jong. La détermination de la présence du $B$. coli dans le lait pasteurisé. Het Algemeen Zuivel en Melkhygienisch weekblade, $21^{\mathrm{e}}$ année, no 6, février 1925, pp. 101-102. Le Lait, 1926, pp. 366.

Etienne. Note sur une modification de la coagulation du lait par le B. coli communis. C. R. Soc. de Biol., 20 janvier 1984.

Gìzes. De la recherche du bacille d'Eberth dans les eaux de boisson. Thèse de Lyon, 1902.

Guerbet. Etude de la réaction du rouge neutre au point de vue chimique. C. R. Soc. de Biol., 1911, p. 514.

H. Kufferath. Contrôle bactériologique et hygiénique des laits. Ann. Inst. Pasteur, 1919, p. 462.

Liacomme. La recherche rapide du coli-bacille dans les eaux de boisson par la méthode du neutral-roth. Journal de Phys, et Path. génér., 1910, p. 90.

Lesage. Contribution à l'étude du B. coli communis. Sem. Méd., nº 6, 1892.

LöHNIs. Précis de Bactériologie agricole et de Technique expérimentale. Traduction française par KUFferath. Lamerlin, éd., Bruxelles, 1921.

Macé. Traité de Bactériologie, 1913, t. II, p. 810.

J. RACE. The Examination of Milk for Public Health Purposes, 1918.

Rochatx. a) Une nouvelle cause d'erreur dans la recherche du coli-bacille en milieu au neutral-roth. Soc. Méd. des Hôpitaux de Lyon, 2 février. Lyon Médical, 21 février 1909.

b) Présence des sucres (lactose, glucose) dans les bouillons du rouge neutre, destiné à la recherche rapide du coli-bacille dans les eaux de boisson. Revue d'Hygiène et de Police sanitaire, t. XXXVI, n 2, 20 février 1914, p. 143.

c) Recherche rapide de la contamination des eaux de boisson. Revue d'Hyg. et de Police sanit., juillet-août 1917, p. 472.

d) Milieux \&े l'esculine et coli-bacilles. C. R. Soc. de Biol., t. LXXXIX, 19 novembre 1923 , p. 1042.

e) La recherche du coli-bacille dans l'eau et dans le lait au moyen du milieu à l'esculine. Le Lait, 1924, p. 541 .

Rochatx et A. Dufourt: a) Coñtribution à l'étude des urobactéries. C. R. de la Soc. de Biologie, 29 octobre 1910.

b) Signification de la réaction du neutral-roth. C. R. Soc. de Biol., 1910, p. 263.

c) Remarques sur la réaction du neutral-roth. C. R. de la Soc. de Biologie, 29 octobre 1910.

Sicre. Au sujet du rouge neutre comme indice du coli-bacille. C. IR. Soc. de Biol., t. LXVI, 1909, p. 152.

W. TANner et C. Dubois-Granvilue. Quelques notes sur l'influence de la chaleur sur les organismes du groupe coli-typhique dans le lait. Le Lait, 1927, p. 287.

TAPERNoux. Industrie et contrôle des produits d'origine animale. (Cours de 1930-31.)

VALLET. Le B. C. dans ses rapports avec le B. typhique. Thèse de Lyon, 1892.

F. Zaykowsiki et E. Staffe. Dégradation des protéines du lait par les bacté. ridies du groupe Coli-typhique (salmonellose). Milchwirtschaftliche Forschungen, t. V, no 3-4, janvier 1928, pp. 361-373. Le Lait, 1930, p. 186. 